\title{
On the Theorem of Wan for $K$-Quasiconformal Hyperbolic Harmonic Self Mappings of the Unit Disk
}

\author{
MilJAN KNEŽEviĆ*
}

\begin{abstract}
We give a new glance to the theorem of Wan (Theorem 1.1) which is related to the hyperbolic bi-Lipschicity of the $K$-quasiconformal, $K \geqslant 1$, hyperbolic harmonic mappings of the unit disk $\mathbb{D}$ onto itself. Especially, if $f$ is such a mapping and $f(0)=0$, we obtained that the following double inequality is valid $2|z| /(K+1) \leqslant|f(z)| \leqslant \sqrt{K}|z|$, whenever $z \in \mathbb{D}$.
\end{abstract}

\section{INTRODUCTION}

Suppose that $\rho$ is a positive function defined and of the class $\mathrm{C}^{2}$ in some subdomain (open and connected) $\Omega$ of the complex plane $\mathbb{C}$ and let $z_{0} \in \Omega$. Recall that the Gaussian curvature of the conformal metric $d s^{2}=\rho(z)|d z|^{2}$ at the point $z_{0}$ is defined as

$$
K_{\rho}\left(z_{0}\right)=-\frac{1}{2} \frac{(\triangle \log \rho)\left(z_{0}\right)}{\rho\left(z_{0}\right)},
$$

where $\triangle$ is the Laplace second order differential operator (the Laplacian). Also, the $\rho$-length of a rectifiable curve $\gamma:[0,1] \rightarrow \Omega$ is given by $|\gamma|_{\rho}=$ $\int_{\gamma} \sqrt{\rho(z)}|d z|$. Otherwise, the $\rho$-distance between the points $z_{1}$ and $z_{2}$ in $\Omega$ is defined as $d_{\rho}\left(z_{1}, z_{2}\right)=\inf |\gamma|_{\rho}$, where the infimum is taken over all rectifiable curves $\gamma$ in $\Omega$ that join the points $z_{1}$ and $z_{2}$.

Example 1.1. Let $\mathbb{D}=\{z \in \mathbb{C}:|z|<1\}$ be the unit disk in $\mathbb{C}$. Consider a conformal metric $d s^{2}=\lambda(z)|d z|^{2}$ on $\mathbb{D}$, where the corresponding density

2010 Mathematics Subject Classification. Primary 30C62, 30C80, 31A05; Secondary 30F15, 30F45.

Key words and phrases. Hyperbolic metric, Harmonic mappings, Quasiconformal mappings.

*Partially supported by the Ministry of Education, Science and Technological Development of the Republic of Serbia, Grant No 174032. 
function $\lambda$ is defined as

$$
\lambda(z)=\left(\frac{2}{1-|z|^{2}}\right)^{2}, z \in \mathbb{D} .
$$

Since, for arbitrary $z \in \mathbb{D}$, we have

$$
\begin{array}{r}
(\triangle \log \lambda)(z)=4(\log \lambda)_{z \bar{z}}(z)=-8\left(\log \left(1-|z|^{2}\right)\right)_{z \bar{z}}(z) \\
=8\left(\frac{\bar{z}}{1-|z|^{2}}\right)_{\bar{z}}(z)=\frac{8}{\left(1-|z|^{2}\right)^{2}},
\end{array}
$$

i.e. $(\triangle \log \lambda)(z)=2 \lambda(z)$, then $K_{\lambda}(z)=-1$, for all $z \in \mathbb{D}$. So, the conformal metric $d s^{2}=\lambda(z)|d z|^{2}$ has the constant and negative Gaussian curvature on $\mathbb{D}$. On the other hand, it is easy to verify that the corresponding distance function induced by this metric on $\mathbb{D}$ is given by the formula

$$
d_{\lambda}\left(z_{1}, z_{2}\right)=\log \frac{1+\left|\frac{z_{1}-z_{2}}{1-z_{2} z_{1}}\right|}{1-\left|\frac{z_{1}-z_{2}}{1-z_{2} z_{1}}\right|}, z_{1}, z_{2} \in \mathbb{D} .
$$

Definition 1.1. The hyperbolic metric on the unit disk is a conformal metric $d s^{2}=\lambda(z)|d z|^{2}$, where the density function $\lambda$ is given by (2). The function $d_{\lambda}$ is called the hyperbolic distance on the unit disk $\mathbb{D}$.

For further properties of the hyperbolic metric we refer to [2] and [9].

Let $\Omega$ and $\Omega^{\prime}$ be some subdomains of the complex plane $\mathbb{C}$.

Definition 1.2. For a mapping $f: \Omega \rightarrow \Omega^{\prime}$, which is of the class $C^{2}$ in $\Omega$, we say that it is harmonic with respect to a conformal metric $d s^{2}=\rho(w)|d w|^{2}$ defined on $\Omega^{\prime}$ ( $\rho$ is a positive function and of the class $C^{2}$ in $\Omega^{\prime}$ ) if

$$
f_{z \bar{z}}(z)+\frac{\rho_{w}(f(z))}{\rho(f(z))} f_{z}(z) f_{\bar{z}}(z)=0,
$$

for all $z \in \Omega$, where $f_{z}$ and $f_{\bar{z}}$ are the partial derivatives of $f$ in $\Omega$ related to the variables $z$ and $\bar{z}$, respectively. Here by $f_{z \bar{z}}$ we denoted the second order partial derivative of the mapping $f$ in $\Omega\left(f_{z \bar{z}}=\left(f_{z}\right)_{\bar{z}}=\left(f_{\bar{z}}\right)_{z}\right)$.

It is obvious that in the presence of the Euclidean metric on the image subdomain $\Omega^{\prime}$, i.e. in the case when the density function $\rho \equiv 0$ on $\Omega^{\prime}$, the relation (4) defines a harmonic function, or Euclidean harmonic mapping, since $(\triangle f)(z)=4 f_{z \bar{z}}(z)=0$, for all $z \in \Omega$.

Definition 1.3. A sense preserving $\mathrm{C}^{1}$ diffeomorphism $f: \Omega \rightarrow \Omega^{\prime}=f(\Omega)$ is said to be regular $K$-quasiconformal (or just $K$-quasiconformal) if there is a constant $K \geqslant 1$ such that $\left|f_{z}(z)\right|^{2}+\left|f_{\bar{z}}(z)\right|^{2} \leqslant \frac{1}{2}\left(K+\frac{1}{K}\right) J_{f}(z)$, for all $z \in \Omega$, where $J_{f}: z \mapsto J_{f}(z)=\left|f_{z}(z)\right|^{2}-\left|f_{\bar{z}}(z)\right|^{2}, z \in \Omega$, is the Jacobian of the mapping $f$. 
Note that if $K=1$ the mapping $f$ is a conformal mapping, since in that case $f_{\bar{z}} \equiv 0$ on $\Omega$.

By using a new approach and technique, in the article [5] we gave a new proof of Wan's result (see [12]) related to the bi-Lipschicity of the quasiconformal hyperbolic harmonic diffeomorphisms of the unit disk. More specifically, we constructed some conformal metrics on the unit disk $\mathbb{D}$ and, by understanding their properties and by calculating their Gaussian curvatures, we applied some versions of the results that are of the Ahlfors-SchwarzPick type to show that every $K$-quasiconformal mapping $f$ of the unit disk $\mathbb{D}$ onto itself, which is also harmonic with respect to the hyperbolic metric $d s^{2}=\lambda(w)|d w|^{2}$ on $\mathbb{D}$, is a quasi-isometry with respect to the hyperbolic metric. Moreover, such a mapping $f$ is $(2 /(K+1), \sqrt{K})$ bi-Lipschitz with respect to the hyperbolic metric, too.

Theorem 1.1 (Wan [12], KM [5]). Let $f \in \mathrm{C}^{2}(\mathbb{D})$ be a $K$-quasiconformal mapping of the unit disk $\mathbb{D}$ onto itself which is harmonic with respect to the hyperbolic metric $d s^{2}=\lambda(w)|d w|^{2}$ on $\mathbb{D}$. Then $f$ is $a(2 /(K+1), \sqrt{K})$ biLipschitz with respect to the hyperbolic metric. Since $\sqrt{K} \leqslant(K+1) / 2$, then $f$ is also a quasi-isometry with respect to the hyperbolic metric.

For details about quasiconformal harmonic mappings we refer a interested reader to [7], [8], [9] and [10].

\section{THE MAIN RESUlT}

Suppose now that a given mapping $f$ satisfies the conditions of the previous theorem. In addition, suppose that $f(0)=0$. According to the Theorem 1.1, we have

$$
d_{\lambda}(f(z), 0) \leqslant \sqrt{K} d_{\lambda}(z, 0) \text { and } d_{\lambda}(f(z), 0) \geqslant \frac{2}{K+1} d_{\lambda}(z, 0),
$$

for all $z \in \mathbb{D}$, and since $d_{\lambda}(r, 0)=\ln \frac{1+r}{1-r}$, for all $0 \leqslant r<1$, we get

$$
|f(z)| \leqslant \frac{(1+|z|)^{\sqrt{K}}-(1-|z|)^{\sqrt{K}}}{(1+|z|)^{\sqrt{K}}+(1-|z|)^{\sqrt{K}}}
$$

and

$$
|f(z)| \geqslant \frac{(1+|z|)^{\frac{2}{K+1}}-(1-|z|)^{\frac{2}{K+1}}}{(1+|z|)^{\frac{2}{K+1}}+(1-|z|)^{\frac{2}{K+1}}}
$$

for all $z \in \mathbb{D}$.

To obtain the main result of this paper, we have to prove the following lemma. 
Lemma 2.1. Let $\alpha>0, \alpha \neq 1$, be a real number. Then the function

$$
a: x \mapsto a(x)=\frac{(1+x)^{\alpha}-(1-x)^{\alpha}}{(1+x)^{\alpha}+(1-x)^{\alpha}}, 0<x<1,
$$

is strictly increasing on the interval $(0,1)$. In addition, if $\alpha>1$, then $a(x)<\alpha x$, for all $0<x<1$, whereas, if $0<\alpha<1$, then $a(x)>\alpha x$, for all $0<x<1$.

Proof. For the defined function $a$ we easily get

$$
a^{\prime}(x)=\frac{4 \alpha\left(1-x^{2}\right)^{\alpha-1}}{\left[(1-x)^{\alpha}+(1+x)^{\alpha}\right]^{2}}>0,
$$

for all $0<x<1$. On the other hand, for its second derivative we have,

$$
a^{\prime \prime}(x)=\frac{8 \alpha\left(1-x^{2}\right)^{\alpha-2}\left[(1+x)^{\alpha}(x-\alpha)+(1-x)^{\alpha}(\alpha+x)\right]}{\left[(1-x)^{\alpha}+(1+x)^{\alpha}\right]^{3}}, 0<x<1 .
$$

Therefore, since for $\alpha>1, \frac{\alpha-x}{\alpha+x}>\frac{1-x}{1+x}>\left(\frac{1-x}{1+x}\right)^{\alpha}$, for all $0<x<1$, we obtain that in this case the function $a$ is concave on $(0,1)$. Otherwise, if $0<\alpha<1$, we have $\frac{\alpha-x}{\alpha+x}<\frac{1-x}{1+x}<\left(\frac{1-x}{1+x}\right)^{\alpha}$, whenever $0<x<1$, and the function $a$ is then convex on $(0,1)$. Now, the statement easily follows from the fact that $a_{+}^{\prime}(0)=\alpha$, where $a_{+}^{\prime}(0)$ is the right derivative of the function $a$ at the point $x=0$.

We are ready now to prove the main result.

Theorem 2.1. Let $f \in \mathrm{C}^{2}(\mathbb{D})$ be a $K$-quasiconformal mapping of the unit disk $\mathbb{D}$ onto itself which is harmonic with respect to the hyperbolic metric $d s^{2}=\lambda(w)|d w|^{2}$ on $\mathbb{D}$. Suppose, in addition, that $f(0)=0$. Then, for all $z \in \mathbb{D}$ we have

$$
\frac{2}{K+1}|z| \leqslant|f(z)| \leqslant \sqrt{K}|z|
$$

for all $z \in \mathbb{D}$.

Proof. The proof is a trivial consequence of the inequalities (5) and (6), and of the Lemma 2.1.

Remark 2.1. In [5] we obtained some version of the Theorem 1.1 that are related to the $K$-quasiconformal harmonic mappings $f$, which are harmonic with respect to some conformal metric defined on the image subdomain, and with the property that its Gaussian curvature is not greater then some negative constant $-a, a>0$. Therefore, we could easily generalize the Theorem 2.1 in this case. 


\title{
REFERENCES
}

[1] L. Ahlfors, Lectures on Quasiconformal Mappings, Van Nostrand Mathematical Studies, D. Van Nostrand, 1966.

[2] L. Ahlfors, Conformal invariants, McGraw-Hill Book Company, 1973.

[3] M. Knežević, Some Properties of Harmonic Quasi-Conformal Mappings, Springer Proceedings in Mathematics and Statistics (LTAPH) 36 (2013), pp. 531-539.

[4] M. Knežević, Kvazikonformna i harmonijska preslikavanja, kvazi-izometrije i krivina, Doktorska disertacija, Univerzitet u Beogradu, Matematički fakultet, Beograd (2014).

[5] M. Knežević, M. Mateljević, On the quasi-isometries of harmonic quasiconformal mappings, Journal Math. Anal. Appl., Vol. 1, No. 334 (2007), pp. 404-413.

[6] O. Lehto, K. I. Virtanen, Quasiconformal Mappings in the Plane, Springer Verlag, 1973.

[7] M. Mateljević, Note on Schwarz lemma, curvature and distance, Zbornik radova PMF 13 (1992), pp. 25-29.

[8] M. Mateljević, Quasiconformality of harmonic mappings between Jordan domains, Filomat, Vol. 3, No. 26 (2012), pp. 479-510.

[9] M. Mateljević, Topics in Conformal, Quasiconformal and Harmonic Maps, Zavod za udžbenike, Beograd (ISBN 978-86-17-17961-6), 2012.

[10] M. Pavlović, Boundary correspondence under harmonic quasi-conformal homeomorfisms of the unit disk, Ann. Acad. Sci. Fenn. Math. 27 (2002) pp. 365-372.

[11] W. Rudin, Real and complex analysis, McGraw-Hill, 1966.

[12] T. Wan, Conastant mean curvature surface, harmonic maps, and univrsal Teichmüller space, J. Diff. Geom. 35 (1992), pp. 643-657.

\author{
Miljan Knežević \\ Faculty of Mathematics \\ Studentski trg 16 \\ 11000 Belgrade \\ Serbia \\ E-mail address: kmiljan@matf.bg.ac.rs
}

